Article

\title{
Model-Based Fault Detection of Inverter-Based Microgrids and a Mathematical Framework to Analyze and Avoid Nuisance Tripping and Blinding Scenarios
}

\author{
Hashim A. Al Hassan, Andrew Reiman, Gregory F. Reed, Zhi-Hong Mao \\ and Brandon M. Grainger* ${ }^{D}$
}

Electric Power Systems Laboratory, Department of Electrical and Computer Engineering, Swanson School of Engineering, University of Pittsburgh, Pittsburgh 15261, PA, USA; haa20@pitt.edu (H.A.A.H.); apr28@pitt.edu (A.R.); gfr3@pitt.edu (G.F.R.); zhm4@pitt.edu (Z.-H.M.)

* Correspondence: bmg10@pitt.edu; Tel.: +412-624-8002

Received: 1 August 2018; Accepted: 15 August 2018; Published: 17 August 2018

\begin{abstract}
Traditional protection methods such as over-current or under-voltage methods are unreliable in inverter-based microgrid applications. This is primarily due to low fault current levels because of power electronic interfaces to the distributed energy resources (DER), and IEEE1547 low-voltage-ride-through (LVRT) requirements for renewables in microgrids. However, when faults occur in a microgrid feeder, system changes occur which manipulate the internal circuit structure altering the system dynamic relationships. This observation establishes the basis for a proposed, novel, model-based, communication-free fault detection technique for inverter-based microgrids. The method can detect faults regardless of the fault current level and the microgrid mode of operation. The approach utilizes fewer measurements to avoid the use of a communication system. Protecting the microgrid without communication channels could lead to blinding (circuit breakers not tripping for faults) or nuisance tripping (tripping incorrectly). However, these events can be avoided with proper system design, specifically with appropriately sized system impedance. Thus, a major contribution of this article is the development of a mathematical framework to analyze and avoid blinding and nuisance tripping scenarios by quantifying the bounds of the proposed fault detection technique. As part of this analysis, the impedance based constraints for microgrid system feeders are included. The performance of the proposed technique is demonstrated in the MATLAB/SIMULINK (MathWorks, Natick, MA, USA) simulation environment on a representative microgrid architecture showing that the proposed technique can detect faults for a wide range of load impedances and fault impedances.
\end{abstract}

Keywords: blinding; fault identification; inverters; microgrids; model-based; nuisance tripping

\section{Introduction}

Protection is one of the most critical and challenging problems when it comes to microgrid systems. Microgrids contain converter-interfaced distributed energy resources (DER) and loads along with traditional electric machinery. In the case of various faults within these architectures, the converters interfacing the DER provide limited fault current in comparison to the response of electric machinery [1-3]. The maximum output current is typically clamped to 1-3 times the rated output current of the converter [4]. This becomes even more problematic if the microgrid were to operate in islanded mode [1-3]. Other reported microgrid concerns that can cause protection difficulty include the bi-directionality of power flow [5], and the diversification of DERs [6,7]. 
When it comes to protecting the microgrid, traditional over-current protection is not a reliable method [5]. A microgrid can experience a fairly wide range of fault current magnitudes and, thus, conventional overcurrent schemes are not effective for microgrid protection [8]. In fact, lower available source currents will result in longer trip times or blinding [6,7]. Fault detection methods utilizing voltage sag information have been successfully demonstrated in the literature [9]. However, microgrid fault detection cannot be based on voltage levels alone [10], because renewables must meet low-voltage and high-voltage ride-through requirements specified in IEEE1547.

Most protection methods proposed in the literature depend on differential protection and a communication system in order to achieve suitable protection metrics which can be slow and very costly $[6,7,11]$. Relying on communication degrades the reliability because the system becomes more prone to a single point of failure. The communication based approaches solve the protection problem by adjusting relay settings through online communication platforms. Examples are found in [12-14] where an extensive communication infrastructure was developed to update the relay settings adaptively or a central supervisory protection unit is utilized. A combination of different protection approaches have been proposed in [15] with differential protection being used as the main strategy. This method is costly and slow since it relies on other mechanisms as backup.

Other approaches make use of advanced signal processing algorithms. Research teams have utilized sequence components [2], which fail in detecting balanced three-phase faults and are unreliable in the case of unbalanced conditions $[7,16]$. Some have used data mining approaches along with the differentials, which are complex methods, and also depend on communication [17]. The same is true for traveling wave-based approaches [18,19]. Wavelet analysis has been proposed in [20] and [21]. In [22], the authors developed a method based on analyzing non-stationary differential signals using the Hilbert Huang transform. This method is complex and suffers from unsatisfactory reliability and high cost due to the differential protection used. The authors in [23] used differential protection as a foundation and tried to improve it by using Hilbert space and fuzzy processes. This method still suffers from the same cost and reliability issues as pure differentials due to the use of differentials. In [24], the authors proposed a solution based on transient polarity comparison, which is based on a wavelet transform but requires a central communication network. Finally, the research team utilizes harmonic impedances to identify faults in [25]. This approach is able to distinguish between high impedance faults and bolted faults.

Other techniques make use of external devices to elevate fault current magnitudes. In [26], the authors have added a flywheel in the microgrid in order to boost fault currents and use traditional over-current protection. Such approaches can be costly and depend on the proper operation of the flywheel. The latest hardware solutions in the literature make use of phasor measurement units $[27,28]$ and a digital central protection unit in [27]. This approach protects radial and looped microgrids against various fault types with the capability of single-phase tripping but still requires communications.

A unique solution that does not fit within the general category of fault identifying techniques listed thus far is found in [29], which makes use of the phase change that is established between measured current and voltage during faults (with loads located on the main bus). With distributed loads (located between the generation and the protection relay) in the system, this method can fail.

In this article a novel, model-based, communication-free fault detection method for inverter-based microgrids is proposed. The method can detect faults in the power transmission paths (feeders) of a microgrid (Figure 1) regardless of the fault current level and the mode of operation (islanded or grid-connected). The developed scheme will overcome the issue of low fault levels that may cause breaker misoperation (nuisance tripping) or prevent the microgrid protection from tripping at all (blinding). It will not use central communication to allow the protection system to operate faster, be more reliable, and be less costly. Another main contribution of this article is the development of a mathematical framework to analyze and avoid blinding and nuisance tripping scenarios. 
The rest of this paper is organized as follows: In the materials and methods section, the microgrid feeder model with different faulted and non-faulted conditions is given along with an explanation of the model-based fault detection approach. In the theory/calculation section, the mathematical proof of the ability to detect faults using one-sided measurement only (communication-less) will be provided and system constraints to avoid nuisance tripping and blinding will be given. In the simulations section, simulation results for the method using two-sided measurement (with communication) and the method using only one-sided measurement (no communication) will be provided. The simulation will demonstrate numerically that the proposed approach is able to distinguish between different conditions (faulted and non-faulted) in an inverter-dominated microgrid that produces low fault current levels. Finally, the article concludes and provides insight into the usefulness of the article with future work described.

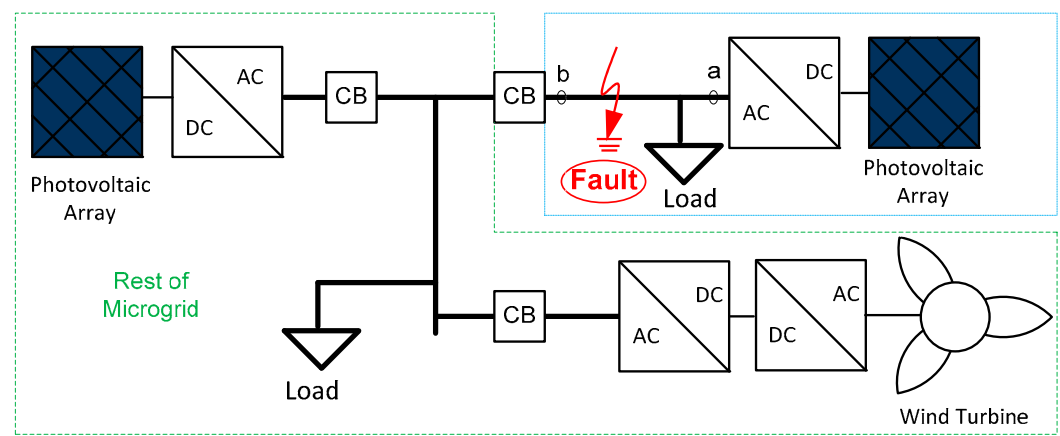

Figure 1. Inverter-based microgrid under study. CB refers to a circuit breaker. The dotted blue line represents a microgrid feeder.

\section{Materials and Methods: Feeder Modelling and Fault Detection Approach}

The major challenge of microgrid protection is that voltage and current levels alone cannot be used to detect faults. Hence, a method based on the structure (internal dynamics) of the microgrid is developed here. This approach is used because the circuit structure will change when a fault occurs within the microgrid. If we can identify the change in the internal system dynamics, we can detect faults. One approach to detect system changes is to have a prior knowledge of the possible system structures modeled as transfer functions, state-space descriptions or static models [30]. Therefore, the different possible mathematical descriptions for different possible faulted conditions and a non-faulted condition must be derived. In the modeling process, the voltage of the system is treated as a system input and the current as a system output. Our methodology for identifying microgrid faults is depicted in Figure 2. The communication-based approach utilizes two measurement locations at both ends of the transmission path (these occur at point $a$ and point $b$ in Figure 1) as inputs to the protection apparatus. The communication-free approach exploits only one measurement located at the microgrid side (point $b$ only in Figure 1) as an input to the protection apparatus. For both approaches, each model produces an estimated current which is compared to the measured current in real-time. When there is a match between a measured current and an estimated current, there will be a clear distinction between a faulted condition and a non-faulted condition. This will lead to the successful detection of a fault despite the low-current levels that the inverter-based sources produce during faults. 


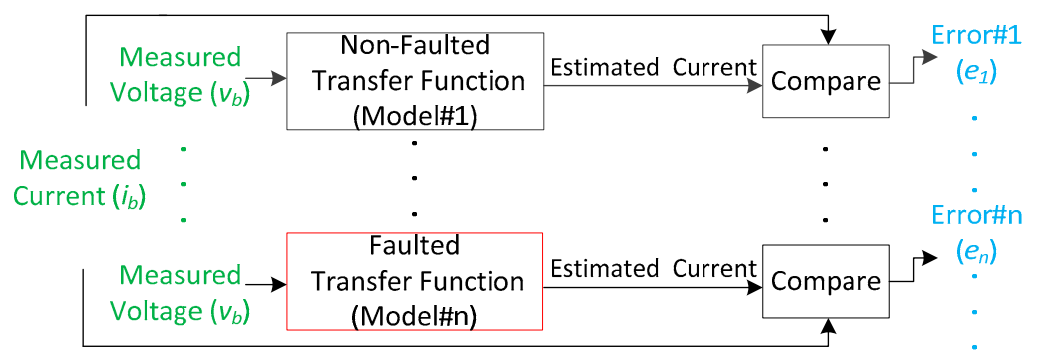

Figure 2. Microgrid fault-detection method.

For the communication-free approach, the error between measured and estimated current could be large. This might hinder the operation of the protection system which can be avoided under certain system constraints derived in forthcoming sections of the article.

A detailed circuit representation of the microgrid feeder of Figure 1 (components within the dotted, blue lined region) is found in Figure 3. The fault locations under evaluation are identified in Figure 3 and shown in red. A fault within the circuit is activated by a switch labeled $S_{f B}, S_{f C}$, or $S_{f D}$. To perform the aforementioned approach, the different models for the non-faulted and faulted microgrid cases are derived in the Appendix A. These models are provided in a state-space format, from which sets of system transfer functions can be obtained, and can be used in the simulation. Three different state-space representations for various fault points within the studied microgrid and one model describing the system under normal operation are derived in the Appendix A to show that there is a clear distinction in the matrices for faulted and non-faulted conditions.

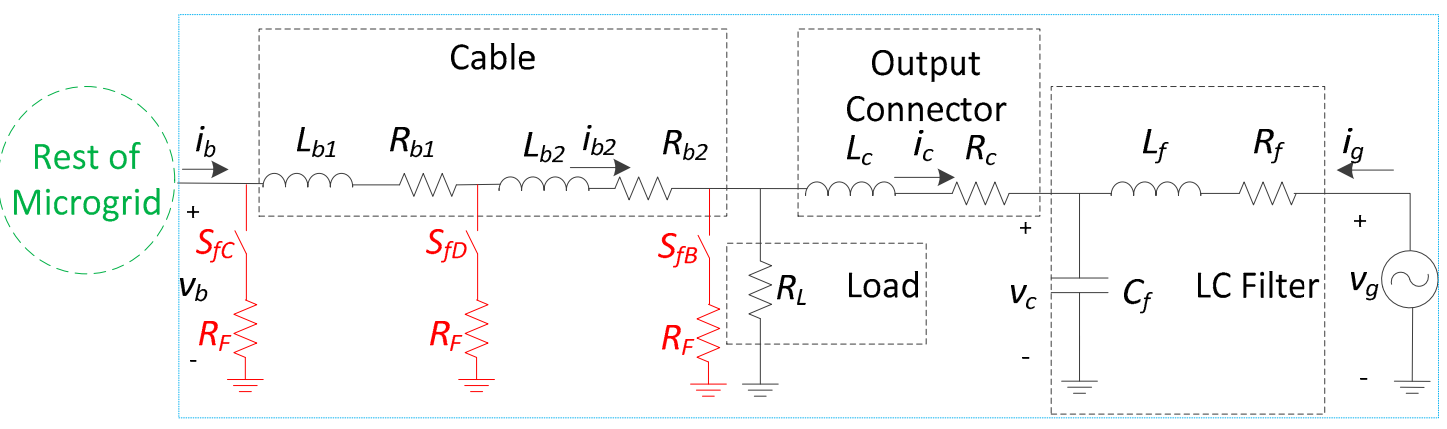

Figure 3. Microgrid feeder circuit during normal operation (switches OPEN) and during faulted conditions (specific switches CLOSED).

If switch $S_{f C}$ is closed, this configuration corresponds to the case of a fault at the beginning of the feeder. If switch $S_{f B}$ is closed, this configuration corresponds to a fault near the system load. If switch $S_{f D}$ is closed, this configuration corresponds to a fault in the middle of the cable. Finally, if all switches are open, this case refers to normal operation of the microgrid.

Note that the system in each condition (non-faulted and faulted conditions) can be represented in the s-domain as

$$
i_{b}(s)=T(s) v_{b}(s)+T_{g}(s) v_{g}(s)
$$

where $T(s)$ is the transfer function resulting from the input $v_{b}$, which can be derived by setting $\rho=0$ (see Appendix A) and solving

$$
T(s)=C(s I-A)^{-1} B+D .
$$

where $T_{g}(s)$ is the transfer function resulting from the input $v_{g}$. The parameters $A, B, C$ and $D$ are matrices corresponding to the state-space description derived in the Appendix A. Four different transfer functions, $T_{n}(s)$ (where $n=1,2,3$ and 4 ) are derived for the four different state space descriptions (see Appendix A) which are used in the fault detection approach described in Figure 2. 


\section{Theory/Calculation: Analytical Analysis of the Communication-Free Approach for Determining System Constraints to Avoid Nuisance Tripping and Blinding}

Relying on communication is not desirable in protection systems because it degrades the reliability, slows protection operation and adds cost. This is why we investigate the effectiveness of a communication-free approach. Hence, we develop a mathematical proof showing that model-based fault detection works when only one measurement from the microgrid side is used under specific constraints.

To do so, we investigate the conditions upon which the fault identification method works when ignoring the source contribution $\left(i_{g}\right)$, filter and output connecter impedance at the end of the feeder. We ignore the source measurement $\left(i_{g}\right.$ and $\left.v_{g}\right)$ because the source and the microgrid side measurements $\left(i_{b}\right.$, and $\left.v_{b}\right)$ are separated by cable with a specific length and would require the use of a communication channel to send the measurement information to the protection system if measurements on both ends are utilized. Hence, by ignoring the source, system fault identification can be communication-free. It is understood that the error between estimated system current and actual current will not be close to zero depending on the available $i_{g}$. However, we propose the following:

Proposition 1. The function giving the smallest error between the actual current and the estimated current describes the state of the system regardless of the size of error and regardless of the magnitude of $i_{g}$ for certain system constraints. This proposition is explained by the inequalities below:

(1) For no fault (all fault switches are opened): $\left|e_{1}\right|<\left|e_{2}\right|$ and $\left|e_{1}\right|<\left|e_{3}\right|$ and $\left|e_{1}\right|<\left|e_{4}\right|$

(2) For ( $S_{f B}$ only closed, other switches opened): $\left|e_{2}\right|<\left|e_{1}\right|$ and $\left|e_{2}\right|<\left|e_{3}\right|$ and $\left|e_{2}\right|<\left|e_{4}\right|$

(3) For ( $S_{f C}$ only closed, other switches opened): $\left|e_{3}\right|<\left|e_{1}\right|$ and $\left|e_{3}\right|<\left|e_{2}\right|$ and $\left|e_{3}\right|<\left|e_{4}\right|$

(4) For ( $S_{f D}$ only closed, other switches opened): $\left|e_{4}\right|<\left|e_{1}\right|$ and $\left|e_{4}\right|<\left|e_{2}\right|$ and $\left|e_{4}\right|<\left|e_{3}\right|$

Note that $e_{n}$ is the error resulting from the difference between the measured value and the estimated output of transfer function $n$ which is derived from condition $n(n=1,2,3$ and 4 for when all switches of Figure 3 are open, $S_{f B}$ is closed, $S_{f C}$ is closed, and $S_{f D}$ is closed, respectively). This error is calculated using the amplitude of the steady-state signal. In equalities 1-4 in Proposition 1 show that the error associated with the transfer function describing the true dynamics of the system must be lower than any of the errors resulting from the remaining transfer functions with incorrect system dynamics for the given microgrid fault scenario in order to successfully detect the fault.

Before proceeding, it is important to note that according to our best knowledge the analysis in this section is a first attempt that analytically examines blinding and nuisance tripping scenarios giving clear indications of when they might occur based on system parameters. This is to allow for protection without the use of communication. Additionally, the analysis provided here presents a general guideline that can be followed to include more faulted scenarios. Hence, this analysis procedure is general enough to be easily extended to $n$ number of faults.

Observing matrices from Equations (A3), (A7), (A8) and (A12) in the Appendix A for the different conditions of the system, we can see that there is a clear distinction between a non-faulted condition shown in Equation (A3) and the faulted conditions shown in Equations (A7), (A8) and (A12). This will be used as a basis to detect faults in the microgrid feeder regardless of fault current levels.

To prove Proposition 1 and find the system constraints guaranteeing its validity, we compare different mathematical relationships describing the system at different scenarios. To do so, we develop a static-based model for the non-faulted and faulted condition of the feeders as shown in Figure 4.

Before proceeding, it is worth noting that the faulted condition used in this analysis is condition $\left(S_{f B}\right.$ closed) only. Other conditions have not been explored because other conditions will give less restrictive constraints. The reason why condition $\left(S_{f B}\right.$ closed) will give the most restrictive constraint is because the current contribution from the DER $\left(i_{g}\right)$ for other conditions $\left(S_{f C}\right.$ closed or $S_{f D}$ closed) will be lower than the current for condition $\left(S_{f B}\right.$ closed). This is because there is more impedance within the path for all 
faulted conditions compared to condition $S_{f B}$. Additionally, the other fault conditions make a significant alteration to the state-space matrix which is evident by inspecting Equations (A3), (A8) and (A12).

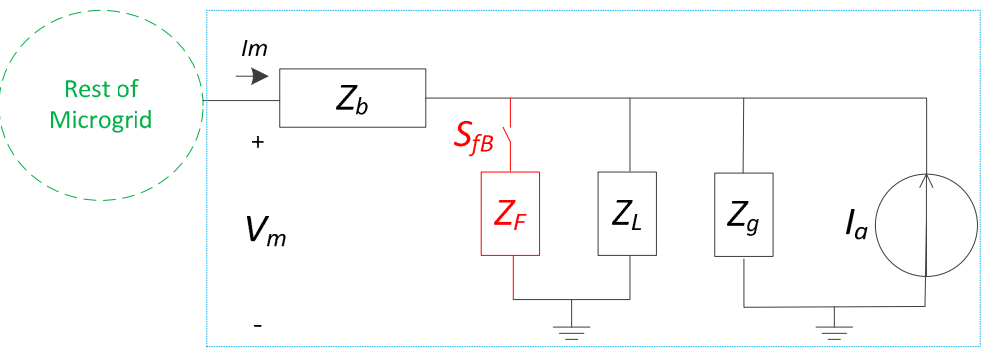

Figure 4. Equivalent impedance-based model for when all switches of Figure 3 are open (normal operation) and during a faulted condition between the load and cable ( $S_{f B}$ closed).

Executive summary of mathematics to prove Proposition 1. Since ignoring the DER-side measurement could lead to detection errors (blinding or nuisance tripping), we investigate through counter-examples on how to avoid these errors. This is done by developing inequality constraints that guarantee the avoidance of those errors (i.e., the counter-examples of blinding and nuisance tripping). We start by assuming that the system is not-faulted and derive equations for the actual measured current (Equation (3)) and of the estimated non-faulted current based upon a one-sided measurement (Equation (4)). Then we assume that the system is faulted and derive the actual measured faulted current (Equation (5)) and the estimated fault current based upon a one-sided measurement (Equation (6)). We then develop the counter examples one by one and develop the constraints as a result of each counter example. Two different counter examples are possible. The first is for the blinding scenario and the second is for a nuisance tripping scenario. The blinding scenario occurs when the system is faulted and the detection device behaves as if the system is healthy. This occurs when the output of Equation (5) is closer in magnitude to that of Equation (4) rather than Equation (6). The nuisance tripping scenario occurs when the system is healthy and the detection device behaves as if the system is faulted. This occurs when the output of Equation (3) is closer in magnitude to that of Equation (6) rather than Equation (4). The formal mathematical relationships of these concepts are derived herewith in. The four inequality constraints listed are derived and guarantee that relay errors do not occur.

Formal mathematics to prove Proposition 1. In Figure 4, the distributed source, filter and output connecter of Figure 3 are lumped into the Norton equivalent represented by $I_{a}$ and $Z_{g}$. Hence, during normal operation (Figure 4) the actual measured value of current magnitude, $\left|I_{m}\right|$, during a non-faulted condition is

$$
\left|I_{m}^{N F}\right|=\left|\frac{\left(V_{m}-I_{a}\left(Z_{L} / / Z_{g}\right)\right)}{Z_{b}+\left(Z_{L} / / Z_{g}\right)}\right|
$$

where $Z_{b}$ is the impedance of the line and $Z_{L}$ is the load impedance. Note that $V_{m}, I_{a}, Z_{L}, Z_{g}, Z_{b}$, are all complex phasors. Note that the magnitude of power phasors are RMS values. In order to prove Proposition 1 and find the constraints upon which the proposition remains valid, we need to find a relationship describing the estimated current for when the current contribution $\left(I_{a}\right)$ from the distributed generation is ignored. This condition is described by (4). Equation (4) is necessary in order to construct inequalities proving Proposition 1 and the reason for its necessity will be explained in further subsections:

$$
\left|I_{E}^{N F}\right|=\left|\frac{V_{m}}{Z_{b}+Z_{L}}\right| .
$$

Here $I_{E}{ }^{N F}$ is defined as the estimate of the measured current for a non-faulted condition, $I_{m}{ }^{N F}$. This estimate of the non-faulted condition could be the output of the transfer function derived from Equations (A3), (A4), (A5) and (A6) if $v_{g}$ is treated as a disturbance (i.e., $\rho=0$ in the relationships found 
in the Appendix A). Throughout this article, we will use the subscript $E$ to denote the value estimated by the model function and subscript $m$ to denote the actual measured value.

For the case when a fault occurs between the load and cable $\left(S_{f B}\right.$ closed in Figure 4$)$ the current magnitude representing the actual measurement is defined by

$$
\left|I_{m}^{F}\right|=\left|\frac{\left(V_{m}-I_{a} Z_{e q}\right)}{Z_{b}+Z_{e q}}\right|
$$

where,

$$
Z_{e q}=Z_{L} / / Z_{F} / / Z_{g} \text {. }
$$

Note that $Z_{e q}$ is a complex phasor, and $Z_{F}=R_{F}$. The current estimate of the faulted condition when $S_{f B}$ is closed is described by

$$
\left|I_{E}^{F}\right|=\left|\frac{V_{m}}{Z_{b}+\left(Z_{L} / / Z_{F}\right)}\right| .
$$

Equations (3)-(6) will be used to prove Proposition 1 under specific system constraints. This is done by disproving all possible counter examples.

\subsection{Theory/Calculation: Analytical Analysis of Masked Fault (Blinding) Scenarios}

The first counter example that will be analyzed here is a masked fault scenario. A masked fault (blinding scenario) is a fault in the system that has not been detected by the proposed, communication-free detection method. When we ignore the contribution of the DER, it is possible to get a masked fault due to the effects of the DER because the DER produces current that we are not accounting for. A masked fault occurs when $\left|e_{1}\right| \leq\left|e_{2}\right|$ while the system is faulted. This scenario occurs when inequality Equation (7) is satisfied:

$$
|| I_{m}^{F}|-| I_{E}^{N F}|| \leq|| I_{m}^{F}|-| I_{E}^{F}|| .
$$

Inequality Equation (7) describes a condition when there is a fault in the system yet the model for the non-faulted case gives a smaller error than the model for the faulted case when compared to actual measurement. This leads to a masked fault condition. Solving Equation (7) gives four distinct regions depending on the left hand side (LHS) and right hand side (RHS) sign of the expressions within the outside absolute value of Equation (7) (LHS $=\left|I_{m}^{F}\right|-\left|I_{E}^{N F}\right|$ and $R H S=\left|I_{m}^{F}\right|-\left|I_{E}^{F}\right|$ ). The four distinct region analyses are as follows:

- $\quad$ If $L H S \geq 0$ and $R H S \geq 0$

Here Equation (7) becomes

$$
\left|I_{m}^{F}\right|-\left|I_{E}^{N F}\right| \leq\left|I_{m}^{F}\right|-\left|I_{E}^{F}\right|
$$

Solving Equation (8) gives

$$
\frac{1}{\left|Z_{b}+Z_{L}\right|} \geq \frac{1}{\left|Z_{b}+Z_{L} / / Z_{F}\right|} .
$$

Note the real and imaginary parts of $Z_{b}, Z_{L}$ and $Z_{F}$ are positive. Therefore, Equation (9) is satisfied when Equation (10) is satisfied.

$$
\left|Z_{L}\right| \leq\left|Z_{L} / / Z_{F}\right|
$$

Inequality Equation (10) can never be satisfied implying that Equation (9) is never satisfied. Thus, Equation (8) is never satisfied. Hence, a masked fault never occurs within this region.

- $\quad$ If $L H S<0$ and $R H S<0$ 
Here Equation (7) becomes

$$
\left|I_{m}^{F}\right|-\left|I_{E}^{N F}\right| \geq\left|I_{m}^{F}\right|-\left|I_{E}^{F}\right|
$$

Inequality Equation (11) is always satisfied because Equation (8) is never satisfied. Therefore, in order to know the conditions of the system giving a masked fault, we check the bounds of this region which is defined by $(L H S<0$ and $R H S<0)$ as

$$
\left|I_{m}^{F}\right|<\left|I_{E}^{N F}\right| \text { and }\left|I_{m}^{F}\right|<\left|I_{E}^{F}\right|
$$

If one of the sides of Equation (12) is dissatisfied then we are guaranteed that Equation (12) is never satisfied because the two sides of Equation (12) have to be satisfied simultaneously. Hence, we only investigate $L H S<0$ which is

$$
\left|I_{m}^{F}\right|<\left|I_{E}^{N F}\right|
$$

Realize that $V_{m}$, and $I_{a}$ are complex quantities, and $\left|I_{m}\right|$ is a the $R M S$ signal. Substituting Equation (4) and Equation (5) into Equation (13), simplifying and rearranging gives

$$
\frac{\left|V_{m}-I_{a} Z_{e q}\right|}{\left|Z_{b}+Z_{e q}\right|}<\frac{\left|V_{m}\right|}{\left|Z_{b}+Z_{L}\right|} .
$$

Our ultimate goal is to disprove all counterexamples or give as few and as relaxed constraints as possible to disprove all counterexamples. For the case if either side of Equation (12) is prevented from being true (never operating in this region), we can guarantee that a masked fault never occurs. Hence, inequality Equation (13) can be made to always be false so that Equation (12) is never satisfied. This is done by performing an inequality reversal to force the system to always operate in the opposite region. Hence, the sign of Equation (14) is redirected and we solve for an impedance ratio limit which is shown as Constraint 1:

Constraint 1: The inequality below must be satisfied to prevent a masked fault scenario:

$$
\frac{\left|V_{m}-I_{a} Z_{e q}\right|}{\left|V_{m}\right|} \geq \frac{\left|Z_{b}+Z_{e q}\right|}{\left|Z_{b}+Z_{L}\right|}
$$

Note that backup protection based on low-voltage (lower than IEEE1547 ride-through requirements) is assumed to always exist. Hence, $V_{m}$ in Constraint 1 is a constant quantity chosen above the backup protection value. Current $I_{a}$ should be as large as possible during faulted conditions which is typically 2 p.u. for inverters. Analyzing the microgrid according to Equation (15), the range of fault impedances $\left(Z_{F}\right)$ that the apparatus can detect can be determined. The constraint should be tested for the minimum and maximum possible values of $Z_{L}$. Constraint 1 represented by Equation (15) provides the following physical insight: the ratio of the faulted system equivalent impedance to the non-faulted system equivalent impedance must be lower than the ratio of the voltage drop across the cable up to the fault point to the total voltage $\left(V_{m}\right)$.

- $\quad$ If $L H S \geq 0$ and $R H S<0$

Here Equation (7) becomes

$$
\left|I_{m}^{F}\right|-\left|I_{E}^{N F}\right| \leq-\left|I_{m}^{F}\right|+\left|I_{E}^{F}\right|
$$

Solving Equation (16) does not give much physical insight. Hence, we reverse the inequality sign of Equation (16) so that it is never satisfied and find a system constraint preventing a masked fault: 
Constraint 2: The inequality below must be satisfied to prevent a masked fault scenario:

$$
\frac{2\left|V_{m}-i_{a} Z_{e q}\right| \cdot\left|Z_{b}+Z_{L}\right|}{\left|V_{m}\right| \cdot\left|Z_{b}+Z_{e q}\right|} \geq \frac{\left|Z_{b}+Z_{L}\right|}{\left|Z_{b}+Z_{L} / / Z_{F}\right|}+1
$$

Note that the result of $Z_{F}$ based on Constraint 1 can be substituted into Constraint 2 to make sure that Equation (17) is true. If it is not, then $Z_{F}$ is lowered and the analysis process is repeated until Equation (17) is satisfied. At the end of the iterations, the maximum value of $Z_{F}$ that the communication-free method can detect will be obtained. An optimization problem can also be formulated to find the optimal values.

- $\quad$ If $L H S<0$ and $R H S \geq 0$

Here Equation (7) becomes

$$
\left|I_{m}^{F}\right|-\left|I_{E}^{N F}\right| \geq-\left|I_{m}^{F}\right|+\left|I_{E}^{F}\right| .
$$

Solving Equation (18) for when $Z_{F}=0 \Omega$ gives us a condition where it is always satisfied. Therefore, we check the system parameters that allow the system to operate in this region if this region exists for a general $Z_{F}$ (all possible types of faults-low and high impedance). This region (LHS $<0$ and $R H S \geq 0$ ) is expressed by

$$
\left|I_{m}^{F}\right|<\left|I_{E}^{N F}\right| \text { and }\left|I_{m}^{F}\right| \geq\left|I_{E}^{F}\right|
$$

By examining Equation (19), we see that the left hand side of this equation is the same as Equation (13). Equation (13) is forced to always be false by Constraint 1. Hence, Equation (19) cannot be satisfied under Constraint 1 . Therefore, a masked fault is impossible for this region.

\subsection{Theory/Calculation: Analytical Analysis of False Positive (Nuisance Tripping) Scenarios}

The only other counterexample that must be disproven to prove Proposition 1 to find all the constraints that guarantee its validity is a false positive (nuisance trip) scenario. A false positive scenario occurs when there is no fault and we falsely identify a fault (i.e., when $\left|e_{2}\right| \leq\left|e_{1}\right|$ while there is no fault). This condition occurs when the following is satisfied:

$$
|| I_{m}^{N F}|-| I_{E}^{F}|| \leq|| I_{m}^{N F}|-| I_{E}^{N F}|| .
$$

Here, the LHS and RHS are redefined to be the expressions within the outside absolute value of (20) (i.e., $L H S=\left|I_{m}^{N F}\right|-\left|I_{E}^{F}\right|$ and $R H S=\left|I_{m}^{N F}\right|-\left|I_{E}^{N F}\right|$ ). Solving Equation (20) gives four distinct regions as follows:

- $\quad$ If $L H S \geq 0$ and $R H S \geq 0$

Here Equation (20) becomes

$$
\left|I_{m}^{N F}\right|-\left|I_{E}^{F}\right| \leq\left|I_{m}^{N F}\right|-\left|I_{E}^{N F}\right|
$$

Solving Equation (21) gives

$$
\frac{1}{\left|Z_{b}+Z_{L} / / Z_{F}\right|} \geq \frac{1}{\left|Z_{b}+Z_{L}\right|}
$$

Equation (22) is the same as Equation (9) but with a reversed inequality sign and we concluded that Equation (9) can never be satisfied. Hence, Equation (22) is always satisfied. Therefore, if this region is entered, there is always a false positive. Hence, we check if it is possible to operate in this 
region or find the conditions that make the system operate within these boundaries. This region is expressed by

$$
\text { LHS } \geq 0 \text { and } R H S \geq 0
$$

Solving LHS $\geq 0$ of (23), we obtain

$$
\frac{\left|V_{M}-I_{a} Z_{e q 1}\right|}{\left|Z_{b}+Z_{e q 1}\right|} \geq \frac{\left|V_{m}\right|}{\left|Z_{b}+Z_{L} / / Z_{F}\right|}
$$

where,

$$
Z_{e q 1}=Z_{L} / / Z_{g} \text {. }
$$

Equation (24) does not give much insight as to whether or not it is possible to operate in this region. Hence, we reverse the inequality sign of Equation (24) so that Equation (24) is never satisfied and find a system constraint (Constraint 3) preventing a false positive. Note that in forthcoming section when we solve for the maximum value of $Z_{F}$, this gives us the value of the highest impedance for a fault on the system, which the apparatus can detect. However, in this section the analysis is for a false positive scenario, which means that there is no fault to begin with. Hence, the constraints here restrict the value of the assumed fault impedance in the model equations. However, when the range of the assumed fault resistance is decreased, this also decreases the range of faults that can be detected when faults occur. Hence, a trade-off between protection system dependability (guaranteeing the relay always operates for faults) and security (guaranteeing the relay will not trip when there is no fault) is made. In protection system design, this trade-off always exists [31] and the same is true for the proposed method. From Constraint 3, to make the protection system more secure, the fault impedance used in the model equations needs to be lowered. However, the more this impedance is lowered, the lower the range of fault impedances that can be detected in the communication-free approach (more security as opposed to more dependability). Hence, a thorough analysis has to be done by the designer to choose whether dependability versus security is valued most for a particular application. It is worth noting here that the communication-based approach is more robust in detecting a wide range of faults with a higher range of fault impedance.

Constraint 3: The fault impedance value used to calculate the equations must be such that the system satisfies the following constraint if security is desired over dependability:

$$
\frac{\left|V_{m}-I_{a} Z_{e q 1}\right|}{\left|V_{m}\right|}<\frac{\left|Z_{b}+Z_{e q 1}\right|}{\left|Z_{b}+Z_{L} / / Z_{F}\right|}
$$

Note that here $V_{m}$ is chosen (for Section 3.2 constraints) based on the largest possible stable normal operating value (i.e., $V_{m}=1.10$ p.u.) as opposed to the smallest possible faulted value for Section 3.1 constraints. This is because a nuisance trip means that we are operating normally as opposed to a faulted condition in a blinding scenario in Section 3.1. Also, the constraint is checked for the largest and smallest possible $I_{a}$ and $Z_{L}$ during normal operation.

- $\quad$ If $L H S<0$ and $R H S<0$

Here (20) becomes

$$
\left|I_{m}^{N F}\right|-\left|I_{E}^{F}\right| \geq\left|I_{m}^{N F}\right|-\left|I_{E}^{N F}\right|
$$

Solving (27) gives

$$
\frac{1}{\left|Z_{b}+Z_{L}\right|} \geq \frac{1}{\left|Z_{b}\right|+\left|Z_{L} / / Z_{F}\right|} .
$$

Inequality (28) is the same as (9) which was proven to never be satisfied in Section 3.1. Hence, (27) is never satisfied and a false positive can never occur in this region. 
- $\quad$ If $L H S \geq 0$ and $R H S<0$

In region ( $L H S \geq 0$ and $R H S \geq 0$ ) Constraint 3 has already been derived to prevent $L H S \geq 0$ from occurring. This means we can never operate in this region under Constraint 3 . Hence, a false positive can never occur in this region.

- $\quad$ If $L H S<0$ and $R H S \geq 0$

Here Equation (20) becomes

$$
\left|I_{m}^{N F}\right|-\left|I_{E}^{F}\right| \geq\left|I_{m}^{N F}\right|+\left|I_{E}^{N F}\right|
$$

Solving Equation (29) yields

$$
2 \frac{\left|V_{m}-I_{a} Z_{e q 1}\right|}{\left|Z_{b}+Z_{e q 1}\right|}-\frac{\left|V_{m}\right|}{\left|Z_{b}+Z_{L} / / Z_{F}\right|} \geq \frac{\left|V_{m}\right|}{\left|Z_{b}+Z_{L}\right|} .
$$

Inspecting Equation (30) does not provide much insight into a system physical constraint. Hence, we investigate the region (LHS $<0$ and $R H S \geq 0$ ) to get insight into the possibility of a false positive.

First, $L H S<0$ is solved to obtain

$$
\frac{\left|V_{m}-I_{a} Z_{e q 1}\right|}{\left|Z_{b}+Z_{e q 1}\right|}<\frac{\left|V_{m}\right|}{\left|Z_{b}+Z_{L} / / Z_{F}\right|} .
$$

Then, $R H S \geq 0$ is solved to achieve

$$
\frac{\left|V_{m}-I_{a} Z_{e q 1}\right|}{\left|Z_{b}+Z_{e q 1}\right|} \geq \frac{\left|V_{m}\right|}{\left|Z_{b}+Z_{L}\right|}
$$

Here, we try to use all possible Equations (30)-(32) to give multiple sufficient conditions guaranteeing the prevention of a false positive. We do this to give more room for $Z_{F}$ to increase in order to increase dependability while keeping the same level of security.

If one of the inequalities of Equations (30), (31) or (32) is reversed then a false positive can never occur. However, Equation (31) cannot be reversed to prevent a false positive because that will violate Constraint 3 developed in Equation (26). Hence, the system must satisfy one of the equations listed as Constraint 4 .

Constraint 4: One of the following conditions must be satisfied to prevent a false positive if security is desired over dependability:

$$
\begin{gathered}
2 \frac{\left|V_{m}-I_{a} Z_{e q 1}\right|}{\left|Z_{b}+Z_{e q 1}\right|}-\frac{\left|V_{m}\right|}{\left|Z_{b}+Z_{L} / / Z_{F}\right|} \leq \frac{\left|V_{m}\right|}{\left|Z_{b}+Z_{L}\right|} . \\
\frac{\left|V_{m}-I_{a} Z_{e q 1}\right|}{\left|V_{m}\right|}<\frac{\left|Z_{b}+Z_{e q 1}\right|}{\left|Z_{b}+Z_{L}\right|} .
\end{gathered}
$$

Note that the values derived from the previous constraints (Constraints 1-3) are checked against Constraint 4 . If Constraint 4 is satisfied, then the analysis procedure ends. If it is not, then the analysis is repeated until all constraints are satisfied.

\section{Results}

Simulation results showing the performance of the proposed approach using communication (two sided measurements) are shown in order to validate the models. The results of the communication-free approach are also shown for different cases. The microgrid here is designed 
based on [32] which satisfies Constraints 1-4. Note that the error values are calculated from the p.u. RMS quantities. The top plots of all the figures demonstrate the effectiveness of the communication-based fault detection approach. The bottom plots of all the figures demonstrate the effectiveness of the communication-free approach proved by the analysis of Section 3.

The top graph of Figure 5 (between $t=0 \mathrm{~s}$ to $t=0.3 \mathrm{~s}$ ) provides a validation of the model developed in the Appendix A for a non-faulted scenario (zero error). After $t=0.3 \mathrm{~s}$, the simulation results in Figures 5-7 provide validations of the faulted models developed in the Appendix A for $\left(S_{f B}\right.$ closed), $\left(S_{f C}\right.$ closed), and $\left(S_{f D}\right.$ closed), respectively, which is clear from the zero error results. The bottom plots demonstrate the validation of the inequalities in Proposition 1 . That is $e_{2}, e_{3}$, and $e_{4}$ should have the lowest errors for Figures 5-7, respectively.

Figure 6 demonstrates the effectiveness of the technique when a load is switched and a fault ( $S_{f C}$ closed) is applied later in the simulation. Figure 8 shows the results when a fault $S_{f D}$ is applied at $20 \%$ of cable length (from load side) with a different fault resistance. This figure shows that the model matches the one described by $\left(S_{f B}\right.$ closed) because this fault model is closest to this condition compared to the other fault model options.

Finally, using the parameters of our microgrid testbed, we swept fault impedances between 0 and $300 \Omega$ and load impedances between 0 and $600 \Omega$ to demonstrate the capability of the detection method. Figure 9 shows the area (detectable space is green) where all the constraints are met (the space where there is no blinding and no nuisance tripping) for fault impedances $\leq 300 \Omega$. This figure shows that the detectable space contains large values of fault impedances and numerically demonstrates the strength of this detection method. This demonstrates that the approach can overcome the distributed load issue in [29].
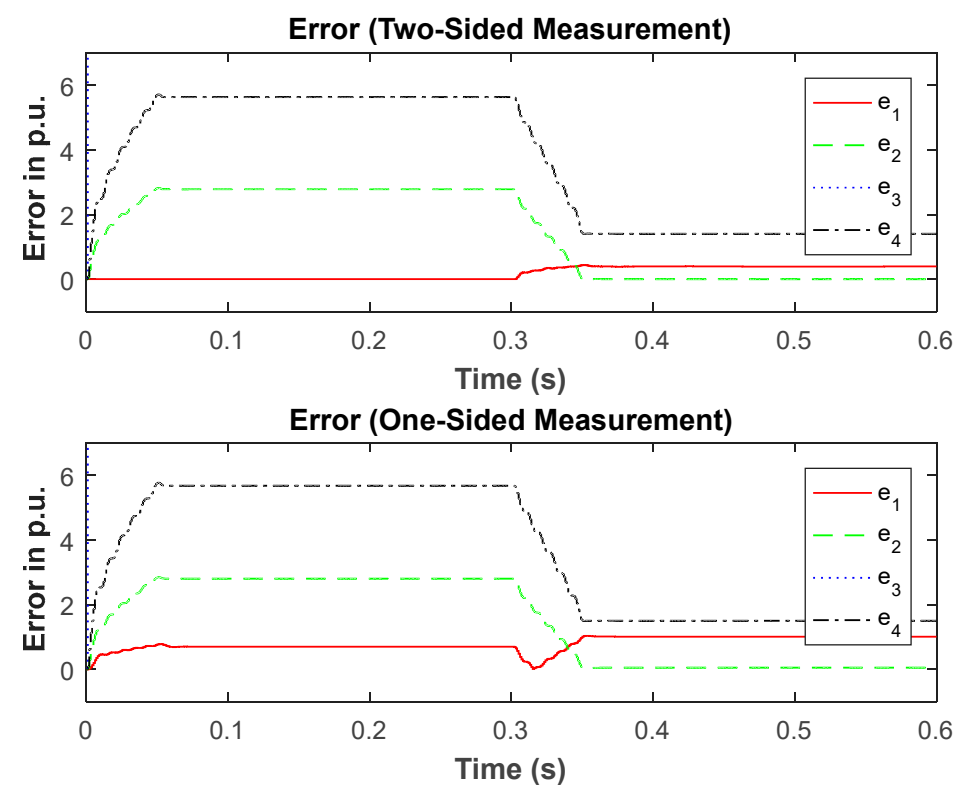

Figure 5. Results during normal operation and when a fault $S_{f B}$ is applied at $0.3 \mathrm{~s}, \mathrm{RF}=0.01 \Omega$. The dotted blue line reaches approx. 82 p.u. before $0.3 \mathrm{~s}$ and approx. 40 p.u. after $0.3 \mathrm{~s}$ (not shown). 

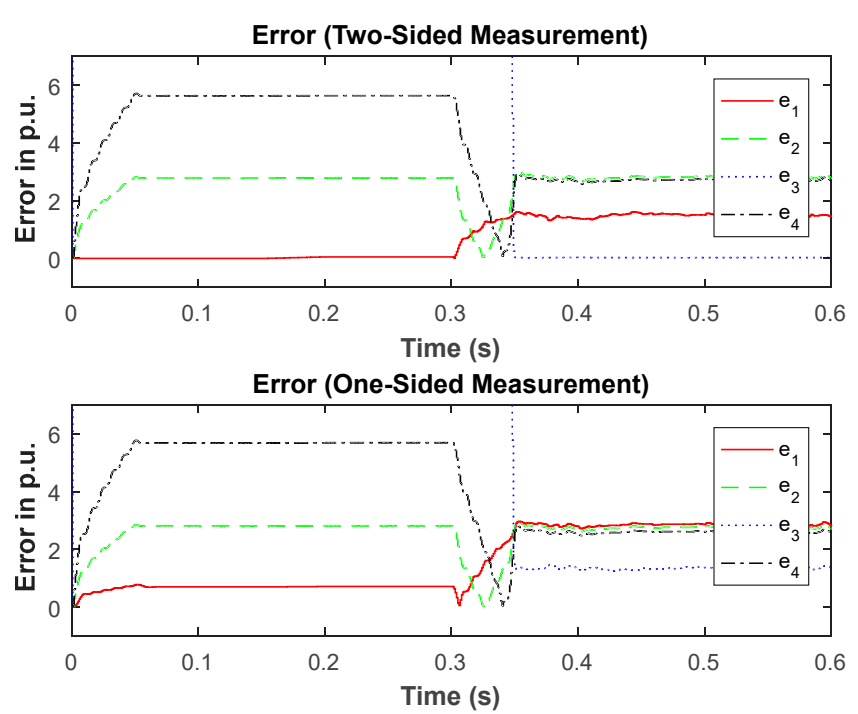

Figure 6. Results when load $\left(\mathrm{RL}=12 \Omega\right.$ ) is switched at $\mathrm{t}=0.15 \mathrm{~s}$ when a fault $S_{f C}$ is applied at $0.3 \mathrm{~s}$, $\mathrm{RF}=0.01 \Omega$. The dotted blue line reaches approx. 82 p.u. before $0.3 \mathrm{~s}$ (not shown).
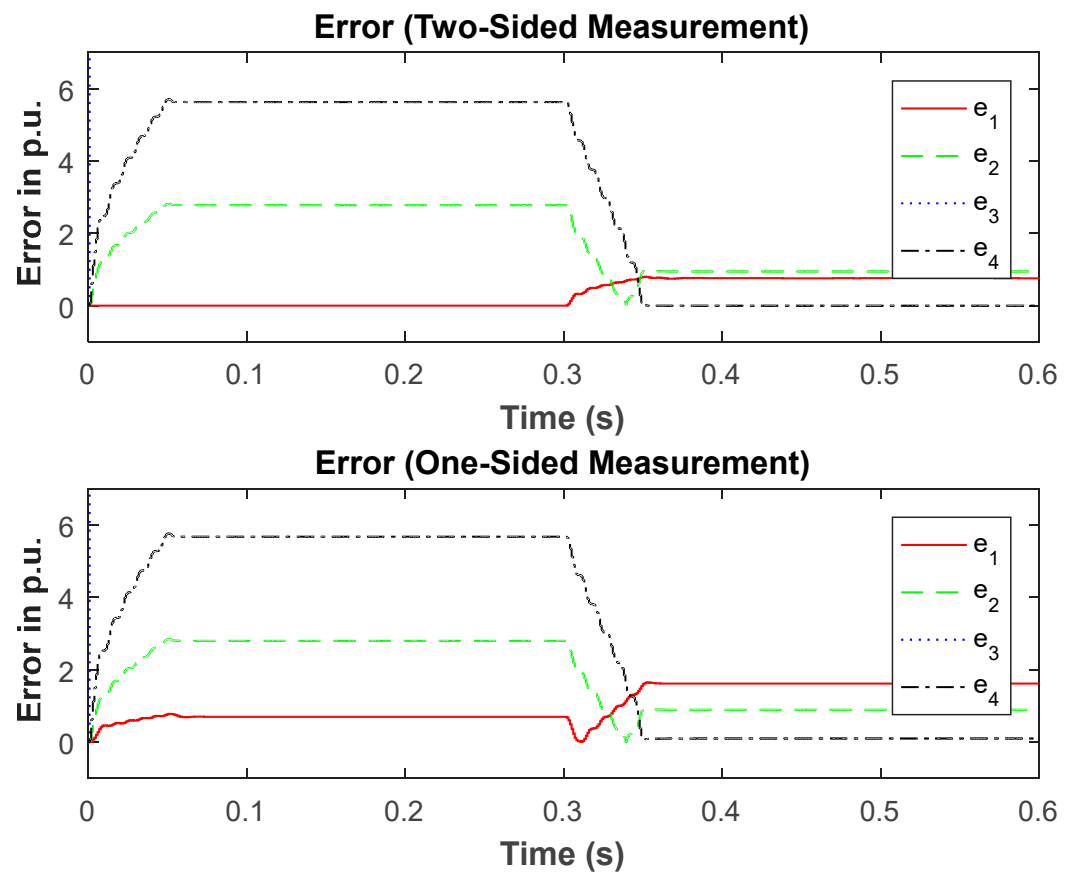

Figure 7. Results during normal operation and when a fault $S_{f D}$ is applied at $0.3 \mathrm{~s}, \mathrm{RF}=0.01 \Omega$. The dotted line reaches approx. 82 p.u. before $0.3 \mathrm{~s}$ and approx. 20 p.u. after $0.3 \mathrm{~s}$ (not shown). 

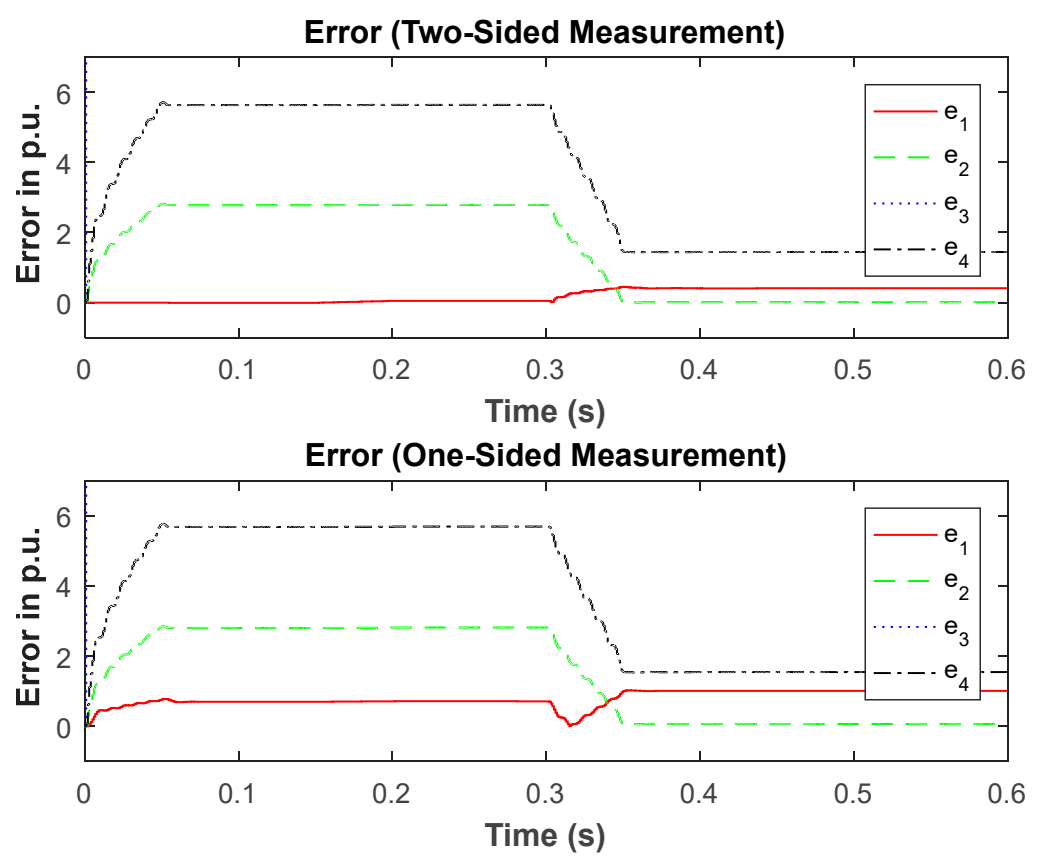

Figure 8. Results when a fault $S_{f D}$ at $20 \%$ of cable length is applied at $0.3 \mathrm{~s}$ with $R F=0.04 \Omega$ after load $(R L=12)$ is switched at $t=0.15 \mathrm{~s}$. The dotted blue line reaches approx. $82 \mathrm{p} . \mathrm{u}$. before $0.3 \mathrm{~s}$ and approx. 40 p.u. after $0.3 \mathrm{~s}$ (not shown).

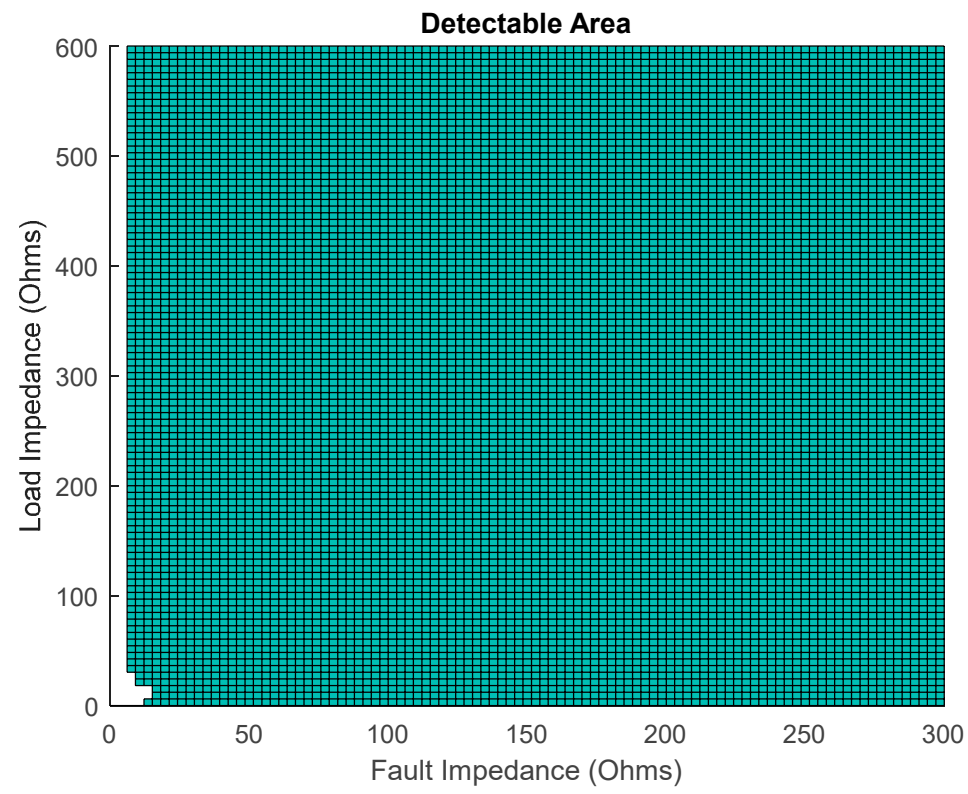

Figure 9. Results for the space where all the constraints are satisfied for fault impedances $\leq 300 \Omega$. The green pixels indicate that the constraints are satisfied (indicating certainty in avoiding blinding and nuisance tripping) and the white pixels indicate the conditions violating any of the constraints (indicating the possibility of nuisance tripping or blinding).

\section{Discussion}

Selecting an appropriate protection scheme for a microgrid design is dependent upon available capital, engineering requirements, and system topology. To compare the model-based approach developed in this work, a qualitatively summary of common relay protection techniques with specific benchmark features is outlined in Table 1. Overcurrent, symmetrical component, overvoltage, 
differential and distance protection schemes are the common routines that are well developed. Adaptive and traveling wave methods have shown promise by various research groups and in test settings. The phase-based and model-based approaches are beginning to be conceptualized and evaluated by the engineering community.

Table 1. Qualitative comparison of general classes of relay protection schemes.

\begin{tabular}{cccccc}
\hline $\begin{array}{c}\text { Relay } \\
\text { Technique }\end{array}$ & Operating Principle & $\begin{array}{c}\text { Robustness Against } \\
\text { Distributed Load? }\end{array}$ & $\begin{array}{c}\text { Communication } \\
\text { System }\end{array}$ & $\begin{array}{c}\text { Handling } \\
\text { Converter Faults }\end{array}$ & Cost \\
\hline Overcurrent & Current & No & Not Required & Weak & Low \\
Symmetrical & Current & Fault Dependent & Not Required & Fair & Moderate \\
Component & Voltage & No & Not Required & Fair & Low \\
Overvoltage & Current & No & Required & Excellent & High \\
Differential & Impedance & No & Not Required & Fair & Moderate \\
Distance & Current & Yes & Required & Excellent & High \\
Adaptive & Current & Yes & Required & Good & High \\
Traveling Wave & Bus Phase & Yes & Required & Excellent & High \\
PMU & No & Not Required & Excellent & Moderate \\
Phase-Based & Voltage \& Current Phase & Yes & Not Required & Excellent & Moderate \\
Model-Based & Impedance Model & This work) & & &
\end{tabular}

With microgrids in mind, the top criteria is understanding if a communication system is required and how robust a protection scheme must be for the distributed loads throughout the system architecture. An interesting observation is that these two criteria often go hand-in-hand, that is, if a communication system exists then the specific method will be robust against distributed loads impacting fault identification. An advantage is shown in that the model-based approach does not require a communication system but is robust enough to identify faults in the presence of distributed load.

The methods requiring communication include differential, adaptive, traveling wave, and PMU approaches. Only the adaptive, traveling wave, and PMU have the required robustness for distributed load but come at a higher cost due to processing power in the hardware. An advantage is found in model-based approaches as they will be cheaper because they require no communication system and are robust against distributed load and varying network impedance as shown in the results section.

Regarding sensing technology, the methods can be ranked. Traveling wave approaches use current sensors, which are a well defined technology. Phase based approaches will need to measure both voltage and current to measure the phase differential changes, which is slightly more challenging than the latter. The model-based work used in this work utilized and understanding of system impedances. An argument can be made that impedance measurement can be challenging to quantify, however, all microgrids are all custom designed and smaller in scale compared to bulk power systems such that the microgrid impedances can be estimated with strong accuracy. Therefore, the model-based approach sensing technology technique becomes comparable to the phase-based approach but the former has higher robustness to fault identification. Adaptive routines use current sensing but the routines require a lot of processing power with the amount of information obtained from the system.

The largest competition for the model-based approach developed in this work are the traveling wave approaches in the context of microgrid design, robustness to distributed loads, sensing technology and cost. However, the main differentiator between both besides cost is the ability to identify blinding and nuisance tripping scenarios. Traveling wave based relay settings require precise tuning for sensitivity reasons and can lead to nuisance tripping. The impedance based constraints for blinding and nuisance tripping scenarios for the model-based approach in this paper have been derived and can be derived similarly for other architectures to ensure blinding and nuisance tripping will not occur. Blinding and nuisance tripping for the advanced, communication based approaches are not analyzed in the literature according to the author's knowledge.

This article provided models for different conditions (faulted and non-faulted) for a microgrid feeder. These models were developed and to be used in a model-based approach to detect faults 
in the case of low-fault current levels in inverter-dominated microgrids. To make the system more reliable, faults can be detected without introducing communication; a mathematical proof and system constraints are provided. The method developed in the article can easily distinguish between non-faulted and faulted conditions of microgrids regardless of fault current levels because faults cause significant alterations to the governing system relationships. The proposed approach can be extended to other microgrid configurations. The faults are detected in steady-state here. However, the models developed in the Appendix A can serve as a basis to develop future methods that operate much faster by investigating the transient region for the model-based fault detection. Additionally, the approach that we followed to derive system constraints provides a general framework that can be applied to other protection methods to avoid nuisance tripping and blinding scenarios. This framework also serves as a general foundation to study DER effects on systems and perform inverter-based system design more effectively.

Author Contributions: Conceptualization, H.A.A.H. and B.G.; methodology, H.A.A.H., A.R., Z.-H.M. and B.G.; software, H.A.A.H.; validation, H.A.A.H., Z.-H.M. and B.G.; formal analysis, H.A.A.H., A.R., Z.-H.M. and B.G.; investigation, H.A.A.H. and B.G.; data curation, H.A.A; writing-original draft preparation, H.A.A.H.; writing-Review \& editing, H.A.A.H., Z.-H.M. and B.G.; visualization, H.A.A.H. and B.G.; supervision, B.G.; project administration, B.G.; funding acquisition, G.F.R. (for Reiman).

Funding: This research received no external funding.

Conflicts of Interest: The authors declare no conflict of interest.

\section{Appendix}

The state-space models of the different conditions of the system are shown here to visually show the clear distinction between faulted and non-faulted conditions.

\section{Appendix A.1 Nominal Feeder Model (Non-Faulted)}

Since our concern is to look at faults along the feeder of the system and not inside the inverter, the inverter and the renewable resource are modeled as a voltage source with filter and an output connector as shown in Figure 3. The dynamics of the microgrid feeder can be modeled in state-space form as in Equations (A1) and (A2).

$$
\begin{aligned}
& \dot{x}=A x+B u \\
& y=C x+D u
\end{aligned}
$$

where the system states are

$$
\begin{aligned}
& x=\left[\begin{array}{lllll}
i_{b} & i_{b 2} & i_{c} & v_{c} & i_{g}
\end{array}\right]^{T} \\
& \equiv\left[\begin{array}{lllll}
x_{1} & x_{2} & x_{3} & x_{4} & x_{5}
\end{array}\right]^{T}
\end{aligned}
$$

and the input and output relationships are defined as

$$
\begin{gathered}
u=\left[\begin{array}{ll}
v_{b} & v_{g}
\end{array}\right]^{T} \\
y=i_{b}=x_{1} .
\end{gathered}
$$

Using circuit laws, the state-space matrices $A, B, C$ and $D$ for the non-faulted condition can be shown to be Equations (A3)-(A6), respectively. Note that $\rho$ is chosen based on the inputs utilized, i.e., $\rho=1$ when there is communication and $\rho=0$ when there is no communication.

$$
A=\left[\begin{array}{ccccc}
-\frac{\left(R_{b}+R_{L}\right)}{L_{b}} & 0 & \frac{R_{L}}{L_{b}} & 0 & 0 \\
0 & 0 & 0 & 0 & 0 \\
\frac{R_{L}}{L_{c}} & 0 & -\frac{\left(R_{c}+R_{L}\right)}{L_{c}} & -\frac{1}{L_{c}} & 0 \\
0 & 0 & \frac{1}{C_{f}} & 0 & \frac{1}{C_{f}} \\
0 & 0 & 0 & -\frac{1}{L_{f}} & -\frac{R_{f}}{L_{f}}
\end{array}\right]
$$




$$
\begin{gathered}
B=\left[\begin{array}{ccccc}
\frac{1}{L_{b}} & 0 & 0 & 0 & 0 \\
0 & 0 & 0 & 0 & \rho \frac{1}{L_{f}}
\end{array}\right]^{T} \\
C=\left[\begin{array}{lllll}
1 & 0 & 0 & 0 & 0
\end{array}\right] \\
D=\left[\begin{array}{ll}
0 & 0
\end{array}\right]
\end{gathered}
$$

where, $R_{b}=R_{b 1}+R_{b 2}$ and $L_{b}=L_{b 1}+L_{b 2}$.

Faulted Condition Model (Fault between Load and Cable):

Consider the case when the fault with impedance, $R_{F}$, occurs near the load within the microgrid depicted in Figure 3. This is when switch $S_{f B}$ is closed. The state-space matrices $A, B, C$ and $D$ for this faulted condition can be shown to be Equations (A4)-(A7), respectively.

$$
A=\left[\begin{array}{ccccc}
\frac{-\left(R_{b}+\gamma\right)}{L_{b}} & 0 & \frac{\gamma}{L_{b}} & 0 & 0 \\
0 & 0 & 0 & 0 & 0 \\
\frac{\gamma}{L_{c}} & 0 & \frac{-\left(R_{c}+\gamma\right)}{L_{c}} & \frac{-1}{L_{c}} & 0 \\
0 & 0 & \frac{1}{C_{f}} & 0 & \frac{1}{C_{f}} \\
0 & 0 & 0 & \frac{-1}{L_{f}} & \frac{-R_{f}}{L_{f}}
\end{array}\right]
$$

where,

$$
\gamma=\frac{R_{L} R_{F}}{R_{L}+R_{F}} .
$$

Appendix A.2 Faulted Condition Model (Fault at Microgrid Side of Cable)

Consider the case when a fault occurs at the end of the feeder closest to the microgrid end of the architecture as shown in Figure 3. That is when $S_{f C}$ is closed. The state-space description for this condition can be expressed by Equations (A8)-(A11):

$$
\begin{gathered}
A=\left[\begin{array}{ccccc}
0 & 0 & 0 & 0 & 0 \\
0 & \frac{-\gamma_{1}}{L_{b}} & \frac{R_{L}}{L_{b}} & 0 & 0 \\
0 & \frac{R_{L}}{L_{c}} & \frac{-\gamma_{2}}{L_{c}} & \frac{-1}{L_{c}} & 0 \\
0 & 0 & \frac{1}{C_{f}} & 0 & \frac{1}{C_{f}} \\
0 & 0 & 0 & \frac{-1}{L_{f}} & \frac{-R_{f}}{L_{f}}
\end{array}\right] \\
B=\left[\begin{array}{ccccc}
0 & \frac{1}{L_{b}} & 0 & 0 & 0 \\
0 & 0 & 0 & 0 & \rho \frac{1}{L_{f}}
\end{array}\right]^{T} \\
C=\left[\begin{array}{lllll}
0 & 1 & 0 & 0 & 0
\end{array}\right] \\
D=\left[\begin{array}{llll}
\frac{1}{R_{F}} & 0
\end{array}\right]
\end{gathered}
$$

where,

$$
\gamma_{1}=R_{L}+R_{b}, \gamma_{2}=R_{c}+R_{L} .
$$

Appendix A.3 Faulted Condition Model (Fault in the Middle of Cable)

This condition corresponds to the scenario when $S_{f D}$ is closed. The state-space description for this scenario is described by Equations (A5), (A6) (A12) and (A13). This covers the wide range of fault locations on a feeder so that one set of dynamic relationships either matches one of the faulted conditions or is closest to one set compared to the others. For instance, when a fault occurs a distance away from the middle of the cable closer to the load, this condition should match the model described 
by Equation (A7) and neither the non-faulted model nor the other faulted models. A simulation case in Figure 8 has been shown to demonstrate that this is true.

$$
\begin{gathered}
A=\left[\begin{array}{ccccc}
\frac{-\gamma_{3}}{L_{b 1}} & \frac{R_{F}}{L_{b 1}} & 0 & 0 & 0 \\
\frac{R_{F}}{L_{b 2}} & \frac{-\gamma_{4}}{L_{b 2}} & \frac{R_{L}}{L_{b 2}} & 0 & 0 \\
0 & \frac{R_{L}}{L_{c}} & \frac{-\gamma_{2}}{L_{c}} & \frac{-1}{L_{c}} & 0 \\
0 & 0 & \frac{1}{C_{f}} & 0 & \frac{1}{C_{f}} \\
0 & 0 & 0 & \frac{-1}{L_{f}} & \frac{-R_{f}}{L_{f}}
\end{array}\right] \\
B=\left[\begin{array}{ccccc}
\frac{1}{L_{b 1}} & 0 & 0 & 0 & 0 \\
0 & 0 & 0 & 0 & \rho \frac{1}{L_{f}}
\end{array}\right]^{T}
\end{gathered}
$$

where,

$$
\gamma_{3}=R_{b 1}+R_{F},
$$

\section{References}

1. Zamani, M.A.; Sidhu, T.S.; Yazdani, A. Investigations into the control and protection of an existing distribution network to operate as a microgrid: A case study. IEEE Trans. Ind. Electron. 2014, 61, 1904-1915. [CrossRef]

2. Mirsaeidi, S.; Said, D.M.; Mustafa, M.W.; Habibuddin, M.H. A protection strategy for micro-grids based on positive-sequence component. IET Renew. Power Gener. 2015, 9, 600-609. [CrossRef]

3. Oureilidis, K.O.; Demoulias, C.S. A fault clearing method in converter-dominated microgrids with conventional protection means. IEEE Trans. Power Electron. 2016, 31, 4628-4640. [CrossRef]

4. Mirsaeidi, S.; Said, D.M.; Mustafa, M.W.; Habibuddin, M.H.; Ghaffari, K. An analytical literature review of the available techniques for the protection of micro-grids. Int. J. Electr. Power Energy Syst. 2014, 58, 300-306. [CrossRef]

5. Lai, K.; Illindala, M.S.; Haj-ahmed, M.A. Comprehensive protection strategy for an islanded microgrid using intelligent relays. In Proceedings of the Industry Applications Society Annual Meeting, Dallas, TX, USA, 18-22 October 2015; pp. 1-11.

6. Nikkhajoei, H.; Lasseter, R.H. Microgrid protection. In Proceedings of the 2007 IEEE Power Engineering Society General Meeting, Tampa, FL, USA, 24-28 June 2007; pp. 1-6.

7. Sortomme, E.; Venkata, S.S.; Mitra, J. Microgrid protection using communication-assisted digital relays. IEEE Trans. Power Deliv. 2010, 25, 2789-2796. [CrossRef]

8. Najy, W.K.; Zeineldin, H.H.; Woon, W.L. Optimal protection coordination for microgrids with grid-connected and islanded capability. IEEE Trans. Ind. Electron. 2013, 60, 1668-1677. [CrossRef]

9. De Santis, M.; Noce, C.; Varilone, P.; Verde, P. Analysis of the origin of measured voltage sags in interconnected networks. Electr. Power Syst. Res. 2018, 154, 391-400. [CrossRef]

10. Elkhatib, M.E.; Ellis, A. Communication-assisted impedance-based microgrid protection scheme. In Proceedings of the 2017 IEEE Power \& Energy Society General Meeting, Chicago, IL, USA, 16-20 July 2017; pp. 1-5.

11. Sortomme, E.; Mapes, G.J;; Foster, B.A.; Venkata, S.S. Fault analysis and protection of a microgrid. In Proceedings of the 2008 40th North American Power Symposium, Calgary, AB, Canada, 8-30 September 2008; pp. 1-6.

12. Brahma, S.M.; Girgis, A.A. Development of adaptive protection scheme for distribution systems with high penetration of distributed generation. IEEE Trans. Power Deliv. 2004, 19, 56-63. [CrossRef]

13. Wan, H.; Li, K.K.; Wong, K.P. An adaptive multiagent approach to protection relay coordination with distributed generators in industrial power distribution system. IEEE Trans. Ind. Appl. 2010, 46, 2118-2124. [CrossRef]

14. Ustun, T.S.; Ozansoy, C.; Ustun, A. Fault current coefficient and time delay assignment for microgrid protection system with central protection unit. IEEE Trans. Power Syst. 2013, 28, 598-606. [CrossRef]

15. Huang, W.; Nengling, T.; Zheng, X.; Fan, C.; Yang, X.; Kirby, B.J. An impedance protection scheme for feeders of active distribution networks. IEEE Trans. Power Deliv. 2014, 29, 1591-1602. [CrossRef]

16. Zamani, M.A.; Sidhu, T.S.; Yazdani, A. A protection strategy and microprocessor-based relay for low-voltage microgrids. IEEE Trans. Power Deliv. 2011, 26, 1873-1883. [CrossRef] 
17. Casagrande, E.; Woon, W.L.; Zeineldin, H.H.; Svetinovic, D. A differential sequence component protection scheme for microgrids with inverter-based distributed generators. IEEE Trans. Smart Grid 2014, 5, 29-37. [CrossRef]

18. Li, X.; Dyśko, A.; Burt, G.M. Traveling wave-based protection scheme for inverter-dominated microgrid using mathematical morphology. IEEE Trans. Smart Grid 2014, 5, 2211-2218. [CrossRef]

19. Li, X.; Dysko, A.; Burt, G. Enhanced protection for inverter dominated microgrid using transient fault information. In Proceedings of the 11th IET International Conference on Developments in Power Systems Protection (DPSP 2012), Birmingham, UK, 23-26 April 2012; p. 20.

20. Shi, S.X.; Jiang, B.; Dong, X.Z.; Bo, Z.Q. Protection of microgrid. In Proceedings of the 10th IET International Conference on Developments in Power System Protection (DPSP 2010), Manchester, UK, 29 March-1 April 2010; p. 11.

21. Cardenas, J.; Muthukrishnan, V.; McGinn, D.; Hunt, R. A hybrid algorithm for fault locating in looped microgrids. In Proceedings of the 2016 IEEE Energy Conversion Congress and Exposition (ECCE), Milwaukee, WI, USA, 18-22 September 2016; pp. 1-6.

22. Gururani, A.; Mohanty, S.R.; Mohanta, J.C. Microgrid protection using Hilbert-Huang transform based-differential scheme. IET Gener. Transm. Distrib. 2016, 10, 3707-3716. [CrossRef]

23. Abdulwahid, A.H.; Wang, S. A new differential protection scheme for microgrid using Hilbert space based power setting and fuzzy decision processes. In Proceedings of the 2016 IEEE 11th Conference on Industrial Electronics and Applications (ICIEA), Hefei, China, 5-7 June 2016; pp. 6-11.

24. Ma, X.; Li, B.; Wang, Q.; Bo, Z.; Zhang, M.; Ma, X. Research on microgrid protection based on transient polarity comparison. In Proceedings of the 2016 China International Conference on Electricity Distribution (CICED), Xi'an, China, 10-12 August 2016; pp. 1-5.

25. Tumilty, R.M.; Brucoli, M.; Burt, G.M.; Green, T.C. Approaches to network protection for inverter dominated electrical distribution systems. In Proceedings of the 3rd IET International Conference on Power Electronics, Machines and Drives (PEMD 2006), Dublin, Ireland, 4-6 April 2006; pp. 622-626.

26. Jayawarna, N.; Jones, C.; Barnes, M.; Jenkins, N. Operating microgrid energy storage control during network faults. In Proceedings of the 2007 IEEE International Conference on System of Systems Engineering, San Antonio, TX, USA, 16-18 April 2007; pp. 1-7.

27. Mirsaeidi, S.; Said, D.M.; Mustafa, M.W.; Habibuddin, M.H.; Ghaffari, K. Fault location and isolation in micro-grids using a digital central protection unit. Renew. Sustain. Energy Rev. 2016, 56, 1-17. [CrossRef]

28. Liu, P.; Wu, Y.; Su, Y.; Duan, B. Fault detection and location of microgrid based on distributed decision. In Proceedings of the IECON 2017-43rd Annual Conference of the IEEE the Industrial Electronics Society, Beijing, China, 29 October-1 November 2017; pp. 5054-5059.

29. Al Hassan, H.A.; Fu, Q.; Bharavaju, V.; Yang, Y.; Grainger, B.M. High-speed algorithm for renewable energy based microgrid fault detection and protective coordination. In Proceedings of the 2017 IEEE Energy Conversion Congress and Exposition (ECCE), Cincinnati, OH, USA, 1-5 October 2017; pp. 519-525.

30. Di Fazio, A.R.; Russo, M.; Valeri, S.; De Santis, M. Linear method for steady-state analysis of radial distribution systems. Int. J. Electr. Power Energy Syst. 2018, 99, 744-755.

31. Horowitz, S.H.; Phadke, A.G. Power System Relaying; John Wiley \& Sons: Chichester, UK, 2008.

32. Bidram, A.; Davoudi, A.; Lewis, F.L.; Guerrero, J.M. Distributed cooperative secondary control of microgrids using feedback linearization. IEEE Trans. Power Syst. 2018, 28, 3462-3470. [CrossRef]

(C) 2018 by the authors. Licensee MDPI, Basel, Switzerland. This article is an open access article distributed under the terms and conditions of the Creative Commons Attribution (CC BY) license (http://creativecommons.org/licenses/by/4.0/). 